COVID-19 AND THE KIDNEYS IN 2020

\section{The COVID-19 pandemic: consequences for nephrology}

\section{Annette Bruchfeld}

The consequences of the COVID-19 pandemic have been devastating; however, evidence suggests that patients with, or at risk of, kidney disease are disproportionally affected. Patients on dialysis and kidney transplant recipients are at higher risk of adverse outcomes from COVID-19, whereas, conversely, patients with severe COVID-19 are at increased risk of acute kidney injury, with short-term and possibly long-term consequences for nephrological care.

2020 has been a challenge for society and health systems worldwide as a result of COVID-19, which was declared a pandemic by the WHO in March 2020. As of 1 November 2020 , the causative virus, SARS-CoV-2, had infected 46 million individuals and caused 1.2 million deaths. Efforts have been invested in the development of mitigation strategies and approaches to manage the acute phase of the illness, as well as in research to provide insights into disease mechanisms. COVID-19 was initially characterized as a febrile respiratory disease but is increasingly recognized as a complex multisystem disease with a wide range of manifestations. Viral injury, uncontrolled inflammation, and the activation of coagulation and complement systems are thought to be important components that underlie disease pathogenesis (FIG. 1).

Early reports, mainly from China, identified underlying chronic kidney disease (CKD) as a risk factor for severe COVID-19 and mortality ${ }^{1}$. A study from New York of over 5,000 individuals with COVID-19 found that age, male sex, heart failure, underlying CKD and a BMI $>40 \mathrm{~kg} / \mathrm{m}^{2}$, were strong predictors of hospital admission and critical illness ${ }^{2}$. The largest study from the UK, which included data from 17 million electronic health records, also identified CKD as a risk factor for mortality in patients with COVID-19, with glomerular filtration rate $(\mathrm{GFR})<30 \mathrm{ml} / \mathrm{min} / 1.73 \mathrm{~m}^{2}$ and organ transplantation conferring a high risk in multivariate analyses ${ }^{3}$. Patients who receive in-centre dialysis have consistently been shown to be at higher risk of community exposure to SARS-CoV-2 infection. A nationwide study of patients on in-centre dialysis from around 1,300 dialysis facilities in the USA reported the seroprevalence of SARS-CoV-2 antibodies to be $3.5-27.2 \%$ - in among the general US population ${ }^{4}$.

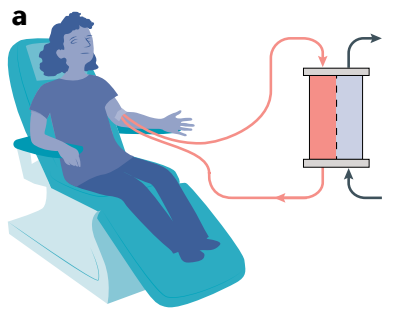

Dialysis and/or transplantation

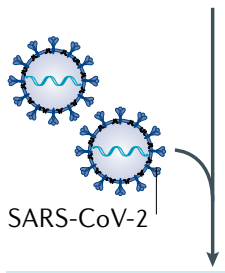

$\uparrow$ SARS-CoV-2 seroprevalence (3.5-27.2\% in patients on dialysis) $\uparrow$ Mortality risk ( $20 \%$ in patients on dialysis and kidney transplant recipients) many places higher than the average of $<10 \%$

COVID-19-associated mortality is also high among dialysis and kidney transplant recipients as demonstrated by a population-based registry study of $>4,000$ patients with a diagnosis of COVID-19 who were receiving kidney replacement therapy $(\mathrm{KRT})^{5}$. Among patients on dialysis $(n=3,285)$, the 28 -day mortality risk was 21.1 times higher than the expected $1.2 \%$ mortality of propensity-score matched historical controls. In transplant recipients $(n=1,013)$, the mortality attributable to COVID-19 was $19.9 \%$ compared with an expected mortality of
The COVID-19 pandemic has had a disproportionate effect on patients at risk of kidney disease

$0.2 \%$ in the matched control group. The study also identified striking differences between age groups: 28 -day mortality among patients women, similar to observations in non-CKD populations. The reason for the sex differences are not fully understood, but hormonal, behavioural and genetic factors have been proposed. Age was likewise an important risk factor for mortality among kidney transplant recipients, evidenced by a mortality of $44.3 \%$ among those aged $>75$ years. Interestingly the mortality risk in this group was higher in women than in men. In both groups, the presence of multimorbidities also affected outcomes. Geographical differences also existed, which are likely associated with the extent of disease spread in different regions ${ }^{5}$.

In addition to the recognition of $\mathrm{CKD}$ as a risk factor for poor outcomes among patients with COVID-19, early evidence also identitant complication of severe COVID-19. Most often, the AKI is mild to moderate, involving a rise in serum creatinine level and signs of kidney damage such as haematuria and on dialysis aged $>75$ years was as high as $31.4 \%$, with a higher mortality risk for men than fied acute kidney injury (AKI) as an impor-

Fig. 1 | The impact of COVID-19 on the kidneys. a | Patients on in-centre dialysis and kidney transplant recipients are at increased risk of community exposure to SARS-CoV-2 infection and COVID-19-associated mortality. $\mathbf{b}$ | Acute kidney injury (AKI) is also an important complication of severe COVID-19, likely as a consequence of multifactorial processes, and is associated with an increased risk of mortality. ICU, intensive care unit. 


\section{Key advances \\ - Underlying chronic kidney disease (CKD) is a risk factor for severe disease and mortality in patients with COVID-19; COVID-19-related mortality is higher in dialysis and transplant recipients than in the general population ${ }^{1-5}$. \\ - Acute kidney injury (AKI) is an important complication of severe COVID-19; critically ill patients in the intensive care unit - particularly those on mechanical ventilation - are more likely than patients with milder disease to require dialysis, which is associated with higher mortality ${ }^{6-8}$. \\ - Most patients who survive COVID-19-associated AKI regain kidney function but up to $30 \%$ may remain on dialysis at discharge $\mathrm{e}^{6-8}$. \\ - Mitigation strategies to reduce exposure to the SARS-CoV-2 virus are vital to protect high-risk CKD populations; whether COVID-19 will increase the prevalence of CKD in the long term and potentially increase the demand for maintenance dialysis is unclear and requires further investigation.}

proteinuria. However, severe cases of AKI that necessitate use of dialysis have been reported, particularly among critically ill patients who are receiving mechanical ventilation; in these patients, AKI is associated with significantly increased mortality. The mechanisms underlying the development of COVID-19associated AKI are likely multifactorial, including viral septicaemia, pneumonia, an enhanced inflammatory response, endothelial damage, hypercoagulability, myocardial dysfunction, drug nephrotoxicity, and the effects of general hypoxia and dehydration on renal perfusion. Tubular and vascular damage have been detected in kidneys of patients with COVID-19, mainly in post-mortem examinations. The extent to which direct or indirect viral tropism is associated with glomerular injury in AKI is not yet resolved, but glomerular pathology has been reported in biopsy samples from patients with COVID-19, including collapsing glomerulopathy, which seems to be associated with the high-risk APOL1 genotype. Several large studies from the USA have identified AKI in up to $50 \%$ of hospitalized patients. One study from New York reported that $20 \%$ of critically ill patients required dialysis and that in-hospital mortality was $50 \%$ among patients with AKI compared with $8 \%$ among those without AKI (adjusted OR 9.2; 95\% CI 7.5-11.3) Another multicentre study of patients from across the USA reported $63 \%$ mortality among patients with dialysis-requiring $\mathrm{AKI}^{7}$. Of patients who survived in the New York study, 30\% had recovered kidney function by the time of discharge from hospital whereas

Whether COVID-19 will escalate the long-term risk of CKD... requires further investigation

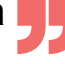

kidney function improved in a further $36 \%$ after discharge ${ }^{6}$. In the multicentre study, one in three patients still required KRT at discharge from hospital, whereas one in six patients remained dialysis-dependent 60 days after admission to the intensive care unit (ICU) ${ }^{7}$. A further study from New York reported an AKI incidence rate of 38.4 per 1,000 patient-days among $>9,600$ patients with COVID-19. Among survivors, $30.6 \%$ remained on dialysis at discharge. Prehospitalization CKD was the only independent risk factor associated with need for dialysis at discharge (adjusted OR 9.3; 95\% CI $2.3-37.8)^{8}$. These findings suggest that most critically ill patients who survive COVID-19 are likely to regain kidney function, but long-term follow-up may be required particularly for patients with prior CKD and in those with lasting signs of kidney injury including haematuria and/or proteinuria.

Only two randomized controlled trials have so far demonstrated beneficial effects of interventional therapies on COVID-19 outcomes. The ACTT- 1 trial demonstrated the antiviral drug remdesivir to be superior to placebo in shortening the time to recovery in adults who were hospitalized with COVID19 and had evidence of lower respiratory tract infection. However, patients with a GFR $<30 \mathrm{ml} / \mathrm{min} / 1.73 \mathrm{~m}^{2}$ were excluded from this study. The large UK RECOVERY trial demonstrated that dexamethasone reduced mortality in hospitalized patients with severe respiratory complications ${ }^{10}$. Although not a primary outcome measure, preliminary data presented at UK Kidney Week 2020 suggested that dexamethasone also reduces the risk of severe AKI needing KRT (R. Haynes, unpublished work).

The COVID-19 pandemic has had a disproportionate effect on patients at risk of kidney disease. Patients with CKD are at high risk of SARS-CoV-2 infection and COVID-19- associated mortality, whereas AKI is a frequent complication of COVID-19 and augments mortality in infected patients, particularly in the ICU setting. Although most patients who survive AKI show improvements in kidney function, their long-term recovery must be evaluated to ensure that nephrology and dialysis resources are allocated accordingly. As new waves of COVID-19 emerge, mitigation strategies to reduce the risk of exposing high-risk populations to the virus remain paramount. Remote care, telemedicine, minimization of blood tests and greater incentives for home dialysis may be helpful in managing patients with CKD, in addition to the implementation of screening, testing and isolation measures in accordance with international guidelines. Whether COVID-19 will escalate the long-term risk of CKD and thereby potentially increase the demand for maintenance dialysis in the future is a concern that requires further investigation.

Annette Bruchfeld ${ }^{1,2}$

'Department of Health, Medicine and Caring Sciences, Linköping University, Linköping, Sweden.

${ }^{2}$ Department of Renal Medicine, Karolinska University Hospital and CLINTEC Karolinska Institutet, Stockholm, Sweden

e-mail: annette.bruchfeld@liu.se https://doi.org/10.1038/s41581-020-00381-4

1. Cheng, Y. et al. Kidney disease is associated with in-hospital death of patients with COVID-19. Kidney Int. 97, 829-838 (2020).

2. Petrilli, C. M. et al. Factors associated with hospital admission and critical illness among 5279 people with coronavirus disease 2019 in New York City: prospective cohort study. BMJ 369, $\mathrm{m} 1966$ (2020).

3. Williamson, E. J. et al. Factors associated with COVID-19-related death using OpenSAFELY. Nature 584, 430-436 (2020).

4. Anand, S. et al. Prevalence of SARS-CoV-2 antibodies in a large nationwide sample of patients on dialysis in the USA: a cross-sectional study. Lancet 396 , 1335-1344 (2020).

5. Jager, K. J. et al. Results from the ERA-EDTA Registry indicate a high mortality due to COVID-19 in dialysis patients and kidney transplant recipients across Europe. Kidney Int. https://doi.org/10.1016/j.kint. 2020.09.006 (2020).

6. Chan, L. et al. AKI in hospitalized patients with COVID-19. J. Am. Soc. Nephrol. https://doi.org/ 10.1681/ASN.2020050615 (2020).

7. Gupta, S. et al. AKI treated with renal replacement therapy in critically ill patients with COVID-19. J. Am. Soc. Nephrol. https://doi.org/10.1681/ ASN. 2020060897 (2020).

8. $\mathrm{Ng}, \mathrm{J}$. H. et al. Outcomes among patients hospitalized with COVID-19 and acute kidney injury. Am. J. Kidney Dis. https://doi.org/10.1053/j.ajkd.2020.09.002 (2020).

9. Beigel, J. H. et al. Remdesivir for the treatment of Covid-19 - final report. N. Engl. J. Med. https:// doi.org/10.1056/NEJMoa2007764 (2020).

10. RECOVERY Collaborative Group. Dexamethasone in hospitalized patients with Covid-19 - preliminary report. N. Engl. J. Med. https://doi.org/10.1056/ NEJMoa2021436 (2020).

\section{Competing interests}

A.B. has received grants and personal fees from AstraZeneca and personal fees from ChemoCentryx, Merck/MSD, Vifor and Abbvie, outside the submitted work. 